\title{
Neural Network Approach for Non-invasive Detection of Hyperglycemia using Electrocardiographic Signals
}

\author{
Linh Lan Nguyen, Steven Su, Member, IEEE, Hung T. Nguyen, Senior Member, IEEE
}

\begin{abstract}
Hyperglycemia or high blood glucose (sugar) level is a common dangerous complication among patients with Type 1 diabetes mellitus (T1DM). Hyperglycemia can cause serious health problems if left untreated such as heart disease, stroke, vision and nerve problems. Based on the electrocardiographic (ECG) parameters, we have identified hyperglycemic and normoglycemic states in T1DM patients. In this study, a classification unit is introduced with the approach of feed forward multi-layer neural network to detect the presences of hyperglycemic/normoglycemic episodes using ECG parameters as inputs. A practical experiment using the real T1DM patients' data sets collected from Department of Health, Government of Western Australia is studied. Experimental results show that proposed ECG parameters contributed significantly to the good performance of hyperglycemia detections in term of sensitivity, specificity and geometric mean $(70.59 \%, 65.38 \%$, and $67.94 \%$, respectively). From these results, it is proved that hyperglycemic events in T1DM can be detected non-invasively and effectively by using ECG signals and ANN approach.
\end{abstract}

\section{INTRODUCTION}

It is well recognized that hyperglycemia is detrimental and one of the risk factors for adverse outcomes in diabetic patients [1]. Persistent hyperglycemia can cause a wide range of chronic complications that affect almost every system in the body and lead to life-threatening conditions, which require immediate medical attention. To detect an increase in blood glucose level, a diabetic patient needs to frequently monitor blood glucose levels by finger prick testing of blood several times a day. This conventional method significantly discourages the patients from periodically checking blood glucose levels. Therefore, there is a strong demand to have a noninvasive technique that helps patients to diagnose the disease easily and painlessly.

New techniques have been employed to develop a minor invasive and/or noninvasive method for blood glucose monitoring, including infrared spectroscopy [2], fluorescence spectroscopy for skin glucose sensing [3], tear glucose [4], etc. However, results from these techniques need checking frequently against direct blood glucose measurements as they cannot replace the direct result of a blood glucose sensor [5]. Although real-time continuous glucose monitoring systems (CGMS) are now available to

Linh Lan Nguyen, Steven Su, and Hung T. Nguyen are with the Centre for Health Technologies, Faculty of Engineering and Information Technology, University of Technology, Sydney, Broadway, NSW 2007, Australia, (e-mail: Linh.L.Nguyen@student.uts.edu.au; Steven.Su@uts.edu.au; Hung.Nguyen@utse.edu.au ) give real-time estimations of glucose levels, there still remain some limitations including time lag and inaccuracy [6]. Due to these limitations, other methods for the detection of abnormal blood glucose levels should be studied.

Among various medical and healthcare information, electrocardiography (ECG) is known to be the best way to measure and diagnose abnormal rhythms of the heart [7]. Several ECG parameters have been investigated to correlate with the prevalence of hyperglycemia. Recently, we have explored significant abnormal changes of ventricular repolarization intervals and certain ECG parameters under hyperglycemia and hypoglycemia conditions that could be good markers to identify hyperglycemia and hypoglycemia in diabetic patients [8]. Reduced heart rate variability has been seen among hyperglycemic patients and our previous study has demonstrated that high blood glucose levels cause significantly lower HRV in subjects with T1DM [9]. Based on these explorations, corresponding ECG parameters could be determined as good markers for classification models to identify hyperglycemic and normoglycemic states in diabetics. However, these findings have not yet been employed to detect hyperglycemia. There are only a few methods to detect hyperglycemia by not using physiological signals, such as in exhaled methyl nitrates [10] and early detection of ongoing $\beta$ cell death [11], which are not commonly used.

Artificial neural network (ANN) has been widely applied in many scientific areas during the past few years, and is currently a very active tool in biomedical research. Most applications of ANNs to biomedical are classification problems; those are extensively utilized in pattern recognition to detect diseases or complex medical conditions. Obviously, ANNs can achieve high performance and computation rates, which are vital in biomedical applications. At least three main studies on hypoglycemia detection, which employ ANNs, have achieved excellent performances with different computational intelligent algorithms [12-14]. Meanwhile, there is no research on hyperglycemia detection using ECG so far and no advanced computational algorithms have been employed for the classification of hyperglycemia.

In this paper, we propose a new non-invasive hyperglycemia detection system, which is based on the approach of ANN and electrocardiographic (ECG) signals in T1DM patients. Section II provides an overview of the method used for non-invasive and continuous detection of hyperglycemia. Section III presents the development and 
results of an optimized neural network to identify hyperglycemia using ECG parameters. A conclusion for this study is shown in section IV.

\section{METHODS}

\section{A. Study}

In T1DM patients, hyperglycemic state is defined as those with more than 1 glucose event $\geq 150 \mathrm{mg} / \mathrm{dl}(8.33 \mathrm{mmol} / \mathrm{l})$ [15]. A database was compiled on the adolescent patients with T1DM who volunteered for overnight hypoglycemia and hyperglycemia study from $9 \mathrm{pm}$ to $6 \mathrm{am}$ at the Princess Margaret Hospital for Children in Perth, Australia. To prevent the interference of hypoglycemic state that may weaken the evaluation of the influence of hyperglycemia in changes of cardiac ventricular intervals, this research only focuses on subjects with normal and high blood glucose levels. Ten type 1 diabetic patients, who mostly had two glycemic states, hyperglycemia $(\mathrm{BGL} \geq 8.33 \mathrm{mmol} / \mathrm{l})$ and normoglycemia $(3.33 \mathrm{mmol} / \mathrm{l}<\mathrm{BGL}<8.33 \mathrm{mml} / \mathrm{l})$, are chosen for collecting data. All data were collected with approval of the Women's and Children's Health Service, Department of Health, Government of Western Australia, and with written informed consent.

18 data points from each patient were used corresponding to the 30-minute duration of each blood glucose sampling point. During the study period, ECG signals were continuously recorded by a medical device called Compumedics with the sampling rate of $512 \mathrm{~Hz}$, while actual blood glucose was collected as reference using Yellow Springs Instruments (Fig. 1).

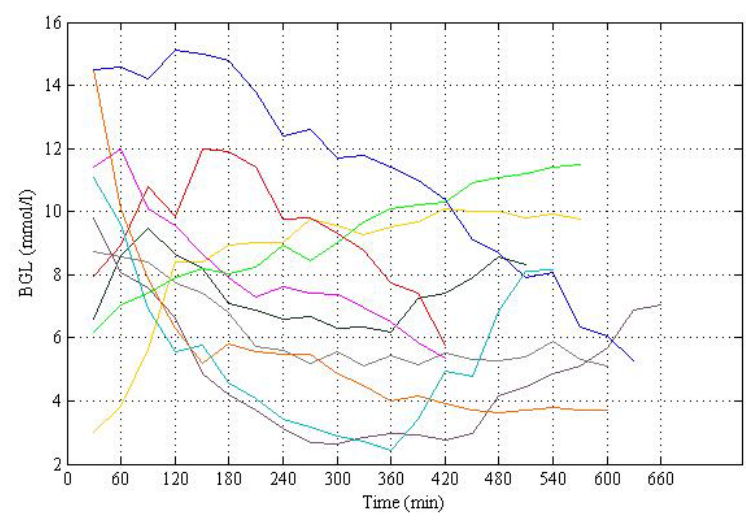

Fig.1. Actual blood glucose levels profiles in $10 \mathrm{~T} 1 \mathrm{DM}$ patients

\section{B. ECG parameters}

30-minute segments of ECG signals were taken according to each sampled blood glucose point for collecting ECG parameters. Based on the effect of hyperglycemia on ECG signals in T1DM, there are sixteen ECG parameters involved to detect hyperglycemia. Five ECG intervals including (i) $\mathrm{HR}$, (ii) $\mathrm{PR}$, (iii) $\mathrm{QT}_{\mathrm{C}}$, (iv) $\mathrm{RT}_{\mathrm{C}}$, (v) $\mathrm{T}_{\mathrm{P}} \mathrm{T}_{\mathrm{EC}}$ were obtained from the patient's ECG signals using a program written in LabVIEW [8]. Index of $c$ in the parameters indicates the correction by heart rate for the variables using the Bazett's formula. The other eleven ECG parameters are a selection of time domain and frequency domain measures of HRV which were considered and performed following the Task Force guidelines [16].

Time domain measures include: (vi) Mean RR interval (MeanRR); (vii) Standard deviation of the RR interval index (SDNN); (viii) Root mean square of successive RR interval differences (RMSSD); (ix) Percentage of consecutive RR intervals that differ by more than $50 \mathrm{~ms}$ (pNN50); (x) HRV triangular index (HRVi); and (xi) Baseline width of the RR interval histogram evaluated through triangular interpolation (TINN). Frequency domain measures involve: (xii) Very low frequency (VLF); (xiii) Low frequency (LF); (xiv) High frequency (HF); (xv) Total spectral power (TotalPw); and (xvi) Ratio between LF and HF components (LF/HF).

HRV analysis was conducted using the Kubios HRV analysis software package (http://kubios.uku.fi/). This software package was used for analyzing the variability of heart beat intervals. It has an easy-to-use graphical interface that shows the HRV waveform and calculates the timedomain, frequency domain, and non-linear dynamics parameters from the raw RR signals. The software computed all required time and frequency domain features.

Independent $t$-test was applied to these features to estimate the significant difference between hyperglycemia and non-hyperglycemia conditions. Statistical analyses were conducted with IBM SPSS version 19 (SPSS Inc, Chicago, IL, USA). Significance value ( $p$-value) less than 0.05 is considered to be significant. Results are presented as mean \pm standard errors.

\section{Classification}

In this study, the detection of hyperglycemic state (BGL $\geq$ $8.33 \mathrm{mmol} / \mathrm{l}$ ) using sixteen ECG variables is based on an advanced neural network algorithm developed from the obtained clinical data. This neural network has a multilayer feed-forward neural network structure with one input layer, one hidden layer and one output layer. Tansig is the hyperbolic tangent sigmoid transfer function used for hidden layer and output layer. To accelerate the convergence of the error back propagation learning method, the ANN is trained by the Levenberg-Marquardt (LM) algorithm. The output layer has one node, which indicates the state of hyper or normo. The overall data set consists of a training set, validation set, and testing set, which are randomly selected with proportions of $35 \%, 35 \%$, and $30 \%$ out of overall data, respectively. The validation set is used as an early stopping method to make sure the ANN does not over-train. The testing set is then used to test the generalization of the neural network. For these, the whole data set, which included both hyperglycemia data part and non-hyperglycemia data part, was used.

To measure the performance of the classification results, sensitivity and specificity are used [17]. The definitions of sensitivity and the specificity are given as follows:

$$
\text { Sensitivity }=\frac{T P}{T P+F N}
$$




$$
\text { Specificity }=\frac{T N}{T N+F P}
$$

where TP (True Positive) is the number of hyperglycemic events which are correctly classified as hyperglycemia; FN (False Negative) is the number of hyperglycemic events which are wrongly classified as normoglycemia; TN (True Negative) is the number of normoglycemic events which are correctly classified as normoglycemia; FP (False Positive) is the number of normoglycemic events which are wrongly classified as hyperglycemia.

For the comparison of the performances, a geometric mean $g m$ was also used. The geometric mean equals to the square root of the multiplication of sensitivity and specificity. The geometric mean is suitable to indicate performance of a detection system with imbalanced data [18]. In our study, the number of normoglycemia data is about double the number of hyperglycemia data. Moreover, four other training methods were also applied to the neural network model to compare their performances with the proposed one. They are Gradient descent, Gradient descent with momentum, Scaled conjugate gradient, Resilient back propagation, which are called LM, GD, GDX, SCG and RP, respectively. The same condition was kept in initializing all the networks. Same input, training parameters and learning function were adopted during training process for those five learning algorithms.

\section{RESULTS AND DISCUSSION}

Normalization was used to reduce patient-to-patient variability and to enable group comparison by subtracting the patient's ECG features to his/her min values and then dividing the results by the differences between the maximum and minimum values [19]. The normalized data will be set in the range between 0 and 1 .

The responses from 10 T1DM patients, as shown in Table I, exhibited significant changes during the hyperglycemic state against normoglycemic state. It can be seen that thirteen out of sixteen parameters in hyperglycemia significantly differed from those in normoglycemia, indicating that a rise in blood glucose levels is associated with a drop in $\mathrm{QT}_{\mathrm{C}}, \mathrm{RT}_{\mathrm{C}}, \mathrm{TpTe}_{\mathrm{C}}$ and $\mathrm{HRV}$ measures.

The neural networks applying five algorithms, namely LM, SCG, GDX, CGF, and RP used all 16 original ECG parameters as inputs. The number of hidden neurons varied between 3 and 20 neurons until the best classification was reached with the minimum mean square error (MSE). Table I shows the best results in terms of sensitivity, specificity and geometric mean from testing set with different input and best numbers of hidden nodes.

LM algorithm, 9 hidden nodes produce the best classification in which the training results of $89.47 \%$ sensitivity and $83.87 \%$ specificity are gained and the testing set leads to $70.59 \%$ sensitivity and $65.38 \%$ specificity. These testing sensitivity and specificity are considered to be a satisfactory result which meets the requirements of disease diagnosis (sensitivity $\geq 70 \%$ and specificity $\geq 50 \%$ ). The best testing geometric mean of $67.94 \%$ by using LM algorithm is also achieved with these sensitivity and specificity. Apart from LM, the sensitivities of the other four algorithms are less than $70 \%$, which are not directed in biomedical application.

TABLE I. Changes Of NORMALIZED 16 ECG PARAMETERS UNDER HYPERGLYCEMIA CONDITIONS COMPARED TO NORMOGLYCEMIC CONDITIONS

\begin{tabular}{cccc}
\hline \hline Parameters & Hyperglycemia & Normoglycemia & $\boldsymbol{p}$ value \\
\hline$H R$ & $0.313 \pm 0.160$ & $0.342 \pm 0.187$ & 0.346 \\
$Q T_{C}$ & $0.438 \pm 0.204$ & $0.536 \pm 0.181$ & $\mathbf{0 . 0 0 3}$ \\
$P R$ & $0.572 \pm 0.229$ & $0.429 \pm 0.229$ & $<\mathbf{0 . 0 0 0 1}$ \\
$R T_{C}$ & $0.433 \pm 0.231$ & $0.552 \pm 0.190$ & $\mathbf{0 . 0 0 1}$ \\
$T p T e_{C}$ & $0.27 \pm 0.167$ & $0.337 \pm 0.185$ & $\mathbf{0 . 0 3}$ \\
$V L F$ & $0.016 \pm 0.018$ & $0.06 \pm 0.129$ & $\mathbf{0 . 0 0 1}$ \\
$L F$ & $0.016 \pm 0.022$ & $0.047 \pm 0.108$ & $\mathbf{0 . 0 0 6}$ \\
$H F$ & $0.077 \pm 0.101$ & $0.151 \pm 0.193$ & $\mathbf{0 . 0 0 2}$ \\
$T o t a l P w$ & $0.032 \pm 0.035$ & $0.080 \pm 0.120$ & $<\mathbf{0 . 0 0 0 1}$ \\
$L F / H F$ & $0.092 \pm 0.103$ & $0.111 \pm 0.167$ & 0.455 \\
$M e a n R R$ & $0.475 \pm 0.245$ & $0.543 \pm 0.2$ & 0.077 \\
$S D N N$ & $0.128 \pm 0.085$ & $0.203 \pm 0.146$ & $<\mathbf{0 . 0 0 0 1}$ \\
$R M S S D$ & $0.220 \pm 0.131$ & $0.303 \pm 0.167$ & $\mathbf{0 . 0 0 2}$ \\
$p N N 50$ & $0.368 \pm 0.211$ & $0.462 \pm 0.189$ & $\mathbf{0 . 0 0 5}$ \\
$H R V i$ & $0.469 \pm 0.167$ & $0.552 \pm 0.176$ & $\mathbf{0 . 0 0 5}$ \\
$T I N N$ & $0.393 \pm 0.176$ & $0.506 \pm 0.197$ & $<\mathbf{0 . 0 0 0 1}$ \\
\hline \hline
\end{tabular}

TABLE II. Best Performances Of Hyperglycemia Detection USING DIFFERENT LEARNING ALGORITHMS

\begin{tabular}{c|c|c|c|c|c|c|c|c|c|c}
\hline \hline \multirow{2}{*}{$\begin{array}{c}\text { Algo- } \\
\text { rithms }\end{array}$} & Hid. & \multicolumn{3}{|c|}{ Training } & \multicolumn{3}{c|}{ Validation } & \multicolumn{3}{c}{ Testing } \\
\cline { 3 - 10 } & nod & $\begin{array}{c}\text { Sen } \\
(\%)\end{array}$ & $\begin{array}{c}\text { Spec } \\
(\%)\end{array}$ & $\begin{array}{c}g m \\
(\%)\end{array}$ & $\begin{array}{c}\text { Sen } \\
(\%)\end{array}$ & $\begin{array}{c}\text { Spec } \\
(\%)\end{array}$ & $\begin{array}{c}\text { gm } \\
(\%)\end{array}$ & $\begin{array}{c}\text { Sen } \\
(\%)\end{array}$ & $\begin{array}{c}\text { Spec } \\
(\%)\end{array}$ & $\begin{array}{c}g m \\
(\%)\end{array}$ \\
\hline$L M$ & 9 & 89.47 & 83.87 & 86.63 & 80 & 77.41 & 78.69 & 70.59 & 65.38 & 67.94 \\
$G D X$ & 12 & 78.57 & 81.82 & 80.18 & 75 & 61.76 & 68.06 & 66.67 & 54.84 & 60.47 \\
$S C G$ & 10 & 72.22 & 68.75 & 70.46 & 90.91 & 53.57 & 69.79 & 68.75 & 59.26 & 63.83 \\
$R P$ & 11 & 88.89 & 68.75 & 78.17 & 72.73 & 75 & 73.86 & 62.5 & 62.96 & 62.73 \\
$C G F$ & 7 & 82.35 & 81.82 & 82.09 & 71.42 & 86.21 & 78.47 & 55.56 & 68 & 61.47 \\
\hline \hline
\end{tabular}

Hyperglycemia detection has been performed with other methods to compare with the performance of the proposed ANN model. Linear Discriminant Analysis (LDA) and KNearest Neighbors (KNN), two of the most conventional and common classification techniques, have been chosen for the comparison. The methods are applied to the same ECG dataset from 10 diabetic patients. LDA and KNN classifiers have been run in training, validation and testing sets with proportions of $35 \%, 35 \%$, and $30 \%$ out of overall data, respectively. The performances have been undertaken by using the Classification Toolbox (version 3.1) in Matlab.

Different numbers of folds for cross-validation have been tested in order to discover the best results in hyperglycemia 
detection. Finally, LDA used 3-fold and KNN used 4-fold cross validation with venetian blinds which achieved the best outcomes, as shown in Table III. Compared to the performance of ANN model using Levenberg-Marquardt for hyperglycemia detection, LDA and KNN still got worse results with lower sensitivities and their sensitivities were much less than their specificities in all three sets of training, validation and testing. The best testing sensibilities of LDA and $\mathrm{KNN}$ are $50 \%$ and $43.75 \%$, which are poorer than the sensibility of ANN (70.59\%). Even Specificities of LDA and KNN are higher than ANN's (77.78\% and $85.19 \%$ vs. $65.38 \%$ ), LDA and KNN do not satisfy with the requirements for disease diagnosis. In general, ANN outperforms LDA and KNN with higher achievement in overall accuracy.

TABle III. COMParison OF Best Performances Of Three DiFFERENT ClASSIFICATION TECHNIQUES FOR HYPERGLYCEMIA DETECTION

\begin{tabular}{c|c|c|c|c|c|c|c|c|c}
\hline \hline \multirow{2}{*}{$\begin{array}{c}\text { Algo- } \\
\text { rithms }\end{array}$} & \multicolumn{3}{|c|}{ Training } & \multicolumn{3}{c|}{ Validation } & \multicolumn{3}{c}{ Testing } \\
\cline { 2 - 10 } & $\begin{array}{c}\text { Sen } \\
(\%)\end{array}$ & $\begin{array}{c}\text { Spec } \\
(\%)\end{array}$ & $\begin{array}{c}\text { gm } \\
(\%)\end{array}$ & $\begin{array}{c}\text { Sen } \\
(\%)\end{array}$ & $\begin{array}{c}\text { Spec } \\
(\%)\end{array}$ & $\begin{array}{c}\text { gm } \\
(\%)\end{array}$ & $\begin{array}{c}\text { Sen } \\
(\%)\end{array}$ & $\begin{array}{c}\text { Spec } \\
(\%)\end{array}$ & $\begin{array}{c}\text { gm } \\
(\%)\end{array}$ \\
\hline ANN & 89.47 & 83.87 & 86.63 & 80 & 77.41 & 78.69 & 70.59 & 65.38 & 67.94 \\
LDA & 73.68 & 84 & 78.61 & 57.14 & 79.31 & 67.32 & 50 & 77.78 & 62.36 \\
$K N N$ & 78.94 & 93.55 & 85.94 & 57.14 & 93.1 & 72.94 & 43.75 & 85.19 & 61.04 \\
\hline \hline
\end{tabular}

For future research, we are continuing to develop advanced algorithms and optimization techniques to improve overall accuracy of hyperglycemia detection. The results of this study might require further validation using different ECG feature extraction methods to yield higher performance. Moreover, a strategy of reducing dimension of input size should be carried out to set up a set of interrelated parameters and find important ECG parameters, which significantly contribute to the performance of hyperglycemia detection. This hyperglycemia detection strategy, after further validation, could be a useful non-invasive and painless method for diabetic patients.

\section{CONCLUSION}

In this paper, a classification unit using artificial neural network is developed to determine the presence of hyperglycemia episodes based on T1DM patients' ECG parameters. The results of $70.59 \%$ sensitivity and $65.58 \%$ specificity by using LM algorithm are considered reasonable and meet the requirement for biomedical application. Various training algorithms also have been developed and compared. In general, the proposed ECG classifier using LM algorithm outperforms other algorithms. This result indicates that hyperglycemic events in T1DM can be detected noninvasively and effectively by using ECG signals and ANN approach.

\section{REFERENCES}

1] Stanley A. Nasraway, "Hyperglycemia During Critical Illness," Journal of Parenteral and Enteral Nutrition, vol. 30, pp. 254-258, MAY-JUNE 2006.

[2] J. J. Burmeister, M. A. Arnold, and G. W. Small, "Noninvasive blood glucose measurements by near-infrared transmission spectroscopy across human tongues," Diabetes Technol Ther, vol. 2, pp. 5-16, 2000.

[3] N. D. Evans, L. Gnudi, O. J. Rolinski, D. J. Birch, and J. C. Pickup, "Non-invasive glucose monitoring by $\mathrm{NAD}(\mathrm{P}) \mathrm{H}$ autofluorescence spectroscopy in fibroblasts and adipocytes: a model for skin glucose sensing," Diabetes Technol Ther, vol. 5, pp. 807-16, 2003.

[4] J. Zhang, W. Hodge, C. Hutnick, and X. Wang, "Noninvasive diagnostic devices for diabetes through measuring tear glucose," $J$ Diabetes Sci Technol, vol. 5, pp. 166-72, 2011.

[5] N. S. Oliver, C. Toumazou, A. E. G. Cass, and D. G. Johnston, "Glucose sensors: a review of current and emerging technology," Diabetic Medicine, vol. 26, pp. 197-210, 2009.

[6] Mark R. Burge, Stephen Mitchell, Alison Sawyer, and David S. Schade, "Continuous Glucose Monitoring: The Future of Diabetes Management," Diabetes Spectrum, vol. 21, pp. 112-119, April 1, 2008.

[7] D.P. Zipes and E. Braunwald, Braunwald's heart disease: a textbook of cardiovascular medicine: W.B. Saunders, 2005.

[8] L. L. Nguyen, S. Su, and H. T. Nguyen, "Identification of hypoglycemia and hyperglycemia in type 1 diabetic patients using ECG parameters," Conf Proc IEEE Eng Med Biol Soc, vol. 9, p. 27162719, 2012.

[9] L. L. Nguyen, S. Su, and H. T. Nguyen, "Effects of hyperglycemia on variability of RR, QT and corrected QT intervals in Type 1 diabetic patients," Conf Proc IEEE Eng Med Biol Soc, vol. 22, p. 1819-1822, 2013.

[10] P. Galassetti. Breath analysis offers potential for non-invasive blood sugar monitoring in diabetes [Online]. Available: http://today.uci.edu/iframe.php?p=/news/release_detail_iframe.asp?ke $\mathrm{y}=1667$

[11] Eitan M. Akirav, Jasmin Lebastchi, Eva M. Galvan, Octavian Henegariu, Michael Akirav, Vitaly Ablamunits, Paul M. Lizardi, and Kevan C. Herold, "Detection of $\beta$ cell death in diabetes using differentially methylated circulating DNA," Proceedings of the National Academy of Sciences, November 9, 2011.

[12] H. T. Nguyen, N. Ghevondian, and T. W. Jones, "Neural-Network Detection of Hypoglycemic Episodes in Children with Type 1 Diabetes using Physiological Parameters," in Engineering in Medicine and Biology Society, 2006. EMBS '06. 28th Annual International Conference of the IEEE, 2006, pp. 6053-6056.

[13] Kit Yan Chan, Sing Ho Ling, T. S. Dillon, and Hung Nguyen, "Classification of hypoglycemic episodes for Type 1 diabetes mellitus based on neural networks," in Evolutionary Computation (CEC), 2010 IEEE Congress on, 2010, pp. 1-5.

[14] Phyo Phyo San, Sai Ho Ling, and H.T. Nguyen, "Optimized variable translation wavelet neural network and its application in hypoglycemia detection system," in Industrial Electronics and Applications (ICIEA), 2012 7th IEEE Conference on, 2012, pp. 547551.

[15] Roman Hovorka, Malgorzata E. Wilinska, Ludovic J. Chassin, and David B. Dunger, "Roadmap to the artificial pancreas," Diabetes research and clinical practice, vol. 74, pp. S178-S182, 2006.

[16] Task Force of the European Society of Cardiology the North American Society of Pacing Electrophysiology, "Heart Rate Variability: Standards of Measurement, Physiological Interpretation, and Clinical Use," Circulation, vol. 93, pp. 1043-1065, March 1, 1996.

[17] D G Altman and J M Bland, "Statistics Notes: Diagnostic tests 1: sensitivity and specificity," BMJ, vol. 308, p. 1552, 1994.

[18] N. Nuryani, S. S. Ling, and H. T. Nguyen, "Electrocardiographic signals and swarm-based support vector machine for hypoglycemia detection," Ann Biomed Eng, vol. 40, pp. 934-45, 2012.

[19] R.L. Kennedy, Solving data mining problems through pattern recognition: Prentice Hall PTR, 1997. 